\title{
Comparative study on the validity of monitoring test indexes of healthy fitness cardiopulmonary track and field sensor
}

Jing Bao ${ }^{1,2^{*}}$

\author{
*Correspondence: \\ baojing@nwu.edu.cn \\ 1 School of Public Policy \\ and Administration, \\ Xi'an Jiaotong University, \\ Xi'an 710049, Shaanxi, China \\ Full list of author information \\ is available at the end of the \\ article
}

\begin{abstract}
With the continuous increase in social pressure and people's higher urgency for physical health, the concept of healthy physical fitness has become more and more important and prominent. However, the research and standards for the validity of the health fitness monitoring test indicators have not been determined. In order to compare the validity of the health fitness test indicators, we adopt the cardiopulmonary track and field sensor technology to monitor the health fitness cardiopulmonary track and field sensor. A comparative study on the validity of test indicators, mainly to test the rationality of the cardiopulmonary endurance evaluation indicators of healthy physical fitness, and to carry out a new design of the concept of healthy physical fitness in track and field teaching, so that it is more inclined to improve the healthy physical fitness of athletes fitness. Research data shows that the average absolute value of the athlete's maximum oxygen consumption is $2.51 \mathrm{~L} / \mathrm{min}$, the highest value is $3.96 \mathrm{~L} / \mathrm{min}$, and the lowest is $2.03 \mathrm{~L} / \mathrm{min}$. The average value of the absolute maximum oxygen consumption of girls is $1.79 \mathrm{~L} / \mathrm{min}$, the highest value is $2.89 \mathrm{~L} / \mathrm{min}$, and the minimum is $1.51 \mathrm{~L} /$ min. From these data, we can know that compared with traditional cardiopulmonary monitoring methods, the sensor monitoring studied in this paper has higher accuracy and wider application range. The peak cardiopulmonary power of athletes' cardiopulmonary function detection using this method is closer to the actual value, while for the peak cardiopulmonary power detected by traditional methods, there is a big difference between the power and the actual value. For different athletes, the advantages of the algorithm in this paper are more obvious, indicating that the method in this paper has a higher detection accuracy for the cardiopulmonary function test of athletes during aerobic training.
\end{abstract}

Keywords: Healthy fitness, Sensor monitoring, Test index validity, Track and field sports

\section{Introduction}

A healthy body is an important part of a healthy physical fitness, which can meet the needs of people in a normal life. Improve the body functions such as the human heart ability, muscle strength and endurance, sensitivity, and body composition indication [1]. Healthy physical fitness is the guarantee for people's normal work and study. People with author(s) and the source, provide a link to the Creative Commons licence, and indicate if changes were made. The images or other third party material in this article are included in the article's Creative Commons licence, unless indicated otherwise in a credit line to the material. If material is not included in the article's Creative Commons licence and your intended use is not permitted by statutory regulation or exceeds the permitted use, you will need to obtain permission directly from the copyright holder. To view a copy of this licence, visit http:// creativecommons.org/licenses/by/4.0/. 
good health and physique can have a good mentality and physique in work, study and entertainment, and are able to deal with people's daily work, leisure and entertainment, and emergencies. Track and field is one of the most popular sports in the world, and it is also the sports with the longest history. Track and field sports are a basic quality exercise program, which can improve muscle endurance and strength, and improve people's cardiorespiratory ability. Track and field sports can be used to improve people's health and physical fitness [2]. However, our track and field sports courses have always ignored the effect of track and field sports on the improvement of human health and fitness. We use the cardiopulmonary track and field sensor to compare the validity of the test indicators of healthy fitness and make it clear that the purpose of fitness and fitness is combined. The training method of track and field sports is to pursue healthy physical fitness and use the best effect of track and field sports.

There are many studies on healthy physical fitness at home and abroad. Among them, Rumi K has studied the difference between physical fitness, namely speed, coordination, agility, explosiveness and other qualities, and healthy physical fitness, and found healthrelated physical fitness. Energy is the physical energy that many students care about preventing diseases, promoting health, improving daily life, learning, and work efficiency [3]. In "Analysis and Comparison of Physical Fitness Concepts and Evaluation Methods in China, the United States, and Japan," Chen W et al. discussed the concepts, evaluation content, and evaluation indicators of physical fitness in China, the USA, and Japan, the basis of physical health, the general problems existing in the test, and the reference for improving the content and methods of national fitness monitoring in my country in the future [4]. Nelis P believes that the step test is reasonable due to its own heart swelling function, and students I have mastered the skills of using this method, and factors such as the gender of the student will have some influence on the experimental results [5].

From the above literature, we can see that although the previous research has relatively sufficient research on physical fitness, the research on the validity of the healthy physical fitness monitoring test index is not sufficient, especially for the physical fitness test combined with the current latest sensor technology, etc. Therefore, the main innovations of this article are (1) the latest cardiopulmonary track and field sensors are used to test and research the health fitness monitoring test indicators, which have higher accuracy. (2) Propose improvements to the traditional athletics training bias, so that the training method is more biased toward the improvement of health and physical fitness. (3) Discuss the rationality of the evaluation indexes of college students' physical fitness cardiopulmonary endurance, and look forward to finding more reasonable test methods and indexes.

\section{Method for monitoring test index validity of healthy physical fitness cardiopulmonary track and field sensor}

\subsection{Concept of physical fitness}

In 1971, the concept of physical fitness was put forward, which means "perform energetically every day without being too tired; enjoy all kinds of leisure time, get enough rest, and be able to cope with emergencies" [6]. With the progress of society with people's emphasis on sports, the concept of physical fitness has become more and more important. It did not appear in Chinese literature until the 1990s and was translated 
by experts from Hong Kong and Taiwan. However, in mainland China, the term began to appear in the twenty-first century [7]. Physical fitness means proper and healthy in English, which is why some scientists regard it as "healthy," that is, physical function is in the most suitable state [8]. There are also German scientists who translated as work ability, French scientists translate it as physical fitness, Japanese scientists translate it as physical strength, and some Hong Kong and Taiwan scientists believe that it is translated as "physical fitness" [9]. In physical fitness, in terms of reasonable meanings and definitions, this research refers to several current editions around the world: "Physical Education and Prospects" defines physical fitness as a person chooses the correct exercise method and amount of exercise under different conditions to improve his physical fitness and maintain health condition, different people should have different levels of exercise due to different physical fitness [10]. The definition of physical fitness in the book "Exploring Modern College Sports" is: physical fitness refers to the need for speed, endurance, and energy exercise and sensitivity. It helps to improve work ability, and the content of exercise includes healthy fitness and technology [11]. The book "Sports and Health" describes physical fitness as: having the spirit of daily activities and good a strong body with a sense of relaxation and emergency response, ordinary exercise will not feel extreme fatigue, but there is no specific description of physical fitness [12]. The World Health Organization describes the concept of physical fitness as: except for daily work, the body will not feel too much tired, while having the power to use for daily entertainment and emergency situations, its content includes healthy physical fitness and technical physical fitness [13]. Hong Kong experts in China define physical fitness as: physical fitness refers to not only suitable for daily work, it is also strong enough to enjoy the joy of life, and the sensitivity and sensitivity of a person who can cope with stress and sudden changes [14]. Physical fitness is a part of the adaptability of the whole body, and it is the spirit and body of people in modern life. The definition of fitness by experts in Taiwan is: First, the body's ability to adapt to health, activities and the environment (such as temperature, climate change, and bacteria). The second is the body's ability to cope with life and adapt to nature. It includes healthy body fitness and competitive fitness. Based on the above six points, the definition, content, and essential description of the term physical fitness are very close to the World Health Organization's definition of physical fitness.

\subsection{Healthy physical fitness and its evaluation index}

In the middle of the twentieth century, the international education system put forward the concept of healthy physical fitness for the first time. Subsequently, some researchers in the USA conducted studies on the differences between exercises related to healthy physical fitness, including explosive power, speed, agility, Sensitivity and other fitness related to healthy physical fitness, and concluded that health physical fitness can prevent diseases, promote health, improve daily life, and enhance learning and efficiency [15]. After consulting-related literature, healthy physical fitness includes four main index components, namely cardiopulmonary fitness, muscle fitness, sensitivity and body composition [16]. Cardiopulmonary capacity: the ability of the lungs, heart, and blood to transport oxygen. Each tissue effectively transports life-sustaining oxygen to various parts of the body, and muscles and cell tissues can effectively use this oxygen for 
metabolism and energy production [17]. Muscle capacity: Muscle strength and muscular endurance are the basic elements of healthy physical fitness. Muscle strength refers to the maximum strength that a muscle can produce when resisting a certain resistance [18]; muscle endurance refers to the number of repetitions and the length of time for continuous muscle exertion in the process of continuous exertion [19]. Sensitivity refers to the range of movement of the limbs and the angle of joint movement. Body composition (appropriate percentage of body fat): Stored fat is more accumulated in the subcutaneous tissue, too much fat causes obesity, and excess fat produced by obesity is a serious health risk factor [20]. At the end of the twentieth century, the American Sports Medicine Association published the American Sports Medicine Monograph in 1992. This book establishes medical tests as a way to check physical fitness. Experimental indicators include $1.6 \mathrm{~km}$ (cardiorespiratory endurance) and push-ups (muscle strength and muscle strength). Healthy physical fitness can keep the body active for a long time, quickly eliminate fatigue and promote physical recovery. Maintaining healthy physical fitness can make people active in sports and recreational activities, and can also allow people to work long hours without excessive fatigue and allows people to exercise in all directions during work and leisure. Healthy physical fitness also has requirements for body composition. Body composition mainly includes fat, muscle, bone, and other relevant parts of the body in an appropriate percentage. Among them, body fat is a key factor in determining body composition, and a healthy body should maintain a certain proportion of body fat. Commonly used diagnostic methods include bioelectrical impedance analysis, two-way X-ray input method, double-labeled water method, ventilation method, skin tightening method, etc. [21]. In view of the above, whether it is in the field of sports science research or the field of medicine, there are several schemes for measuring specific indicators of health and fitness. Also, due to regional and ethnic differences, the selected indicator systems have substantial differences. In the actual situation, even if there are many test items, it is impossible to comprehensively, objectively and scientifically evaluate the individual's physical fitness [22]. Based on the selection of traditional healthy physical fitness evaluation indicators, this study combined with novel and effective cardiopulmonary track and field sensors to conduct related research to observe the changes in the limbs and heart function reserve of different subjects during exercise, with a view to healthy physical fitness Evaluation provides novel, comprehensive and effective evaluation indicators, and provides a more comprehensive, scientific and efficient method and guidance.

\subsection{Research optimal configuration of physical fitness cardiopulmonary track and field sensors based on intelligent algorithms}

With the development of sensor technology, low-energy electronics, and radio frequency technology, low-energy wireless microelectronic sensors are gradually being used on a large scale, and a responsive wireless sensor network is born. Due to the characteristics of intelligent perception, small size, light weight, accurate data, good real-time performance, and no errors in the motion sensor monitoring technology in track and field events, the test results can be objectively fed back in time when the athlete's proprioception has not disappeared. The purpose of improving exercise technology and monitoring exercise effects, thereby improving the effectiveness and pertinence of training, 
reducing blindness, excessive fatigue and injury during exercise, and improving athletic performance.

Based on the health perception of the Internet of Things and the flexible resources of intelligent algorithms, it realizes the collection and management of sports personnel health information, community physical examination, community diagnosis and other information, and integrates personal health perception equipment data, medical data, hospital diagnosis and treatment information, and various types of information. Healthrelated data, so as to establish residents' sports health files, provide residents with personal-centered sports health services, and realize remote diagnosis, consultation, and corresponding remote medical services of sports personnel's health information, and realize cloud-based sports personnel health information management and services [23]. The data flow diagram of the system is shown in Fig. 1.

In the sensor subsystem, various sensors are installed to sense the loads and effects of moving people in various environments, and they are stored in the computer in a certain way [24]. The sensor is a certain Wu Lilian measuring device that converts the measurement to a certain degree with a certain degree of correspondence, and is convenient to use. It usually consists of a sensitive element and a conversion element to form a conversion circuit. The conversion element takes the input of the sensitive element as the output, and converts the input into circuit parameters. The sensitive element can directly sense the measured and output a certain physical quantity element that has a certain relationship

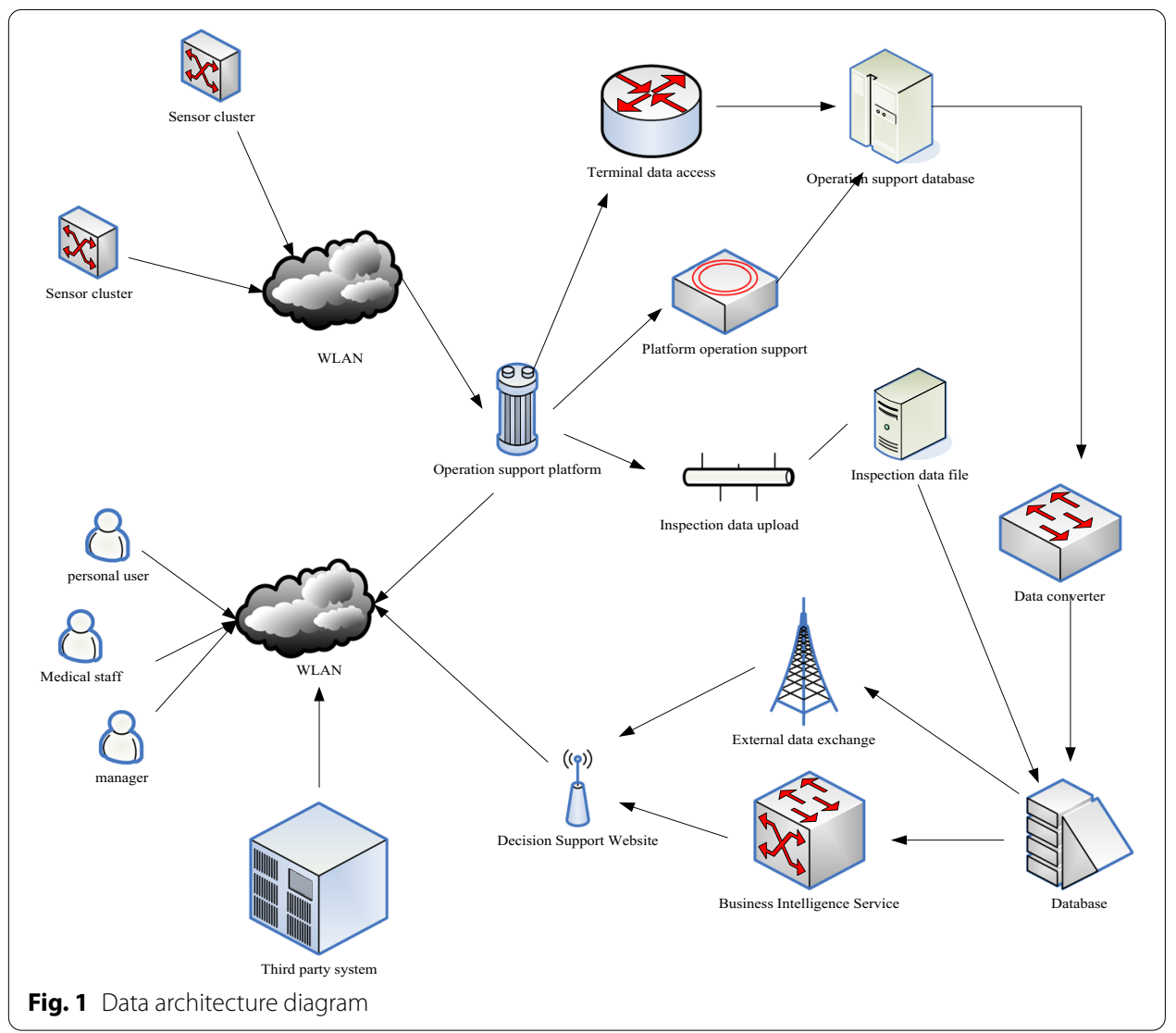


with the measured. The data processing and control subsystem is used to comprehensively control data collection, transmission, processing, storage and display, and can assist in the process of calibration, processing, transmission and reliability testing of the data acquired by the data acquisition and transmission subsystem key data for subsequent status assessment. The data management and analysis evaluation subsystem mainly includes functions such as monitoring data management, structural state early warning, reliability evaluation, fatigue evaluation, and comprehensive structural state evaluation. The relationship between the various subsystems is shown in Fig. 2.

How to make the information of the measured person measured by the sensor can most truly reflect the current person's health status, and realize the evaluation of the person's status is a key research issue of sensor optimization configuration [25]. Before constructing the optimal sensor configuration model, we must first establish a finite element model of human health, perform modal analysis, and obtain the modal vibration matrix $\Psi$ according to the modal data obtained from the modal analysis [26, 27]. According to the characteristics of the sensor optimal configuration problem, construct the objective function for:

$$
\min \max \left\{\mathrm{MAC}_{m n}\right\}
$$

$$
\operatorname{MAC}_{m n}=\frac{\left(\psi_{m} \psi_{n}\right)^{2}}{\left(\psi_{m} \psi_{m}\right)\left(\psi_{n} \psi_{n}\right)}
$$

$\Psi_{m}$ is the mode vector of the $m$ th order; $\Psi_{n}$ is the mode vector of the $n$th mode. If the non-diagonal element $\mathrm{MAC}_{m n}$ of the MAC matrix approaches 0 , it indicates that the $m$ th and $n$th modes have better resolvability, and the linear independence is good; on the contrary, the resolution is not good. As a typical combinatorial optimization problem, the optimal configuration of healthy physical fitness cardiopulmonary track and field sensors has high computational complexity. The currently developed sensor configuration methods can be roughly divided into traditional optimization algorithms and intelligent optimization algorithms $[28,29]$. The traditional optimization algorithm assumes that the modal vectors in the modal matrix $\Psi$ of the theoretical model are independent and orthogonal, and the output of initially selecting $\times$ candidate measuring points is:

$$
S=\psi_{x} p
$$

The estimated simulation coordinates are:

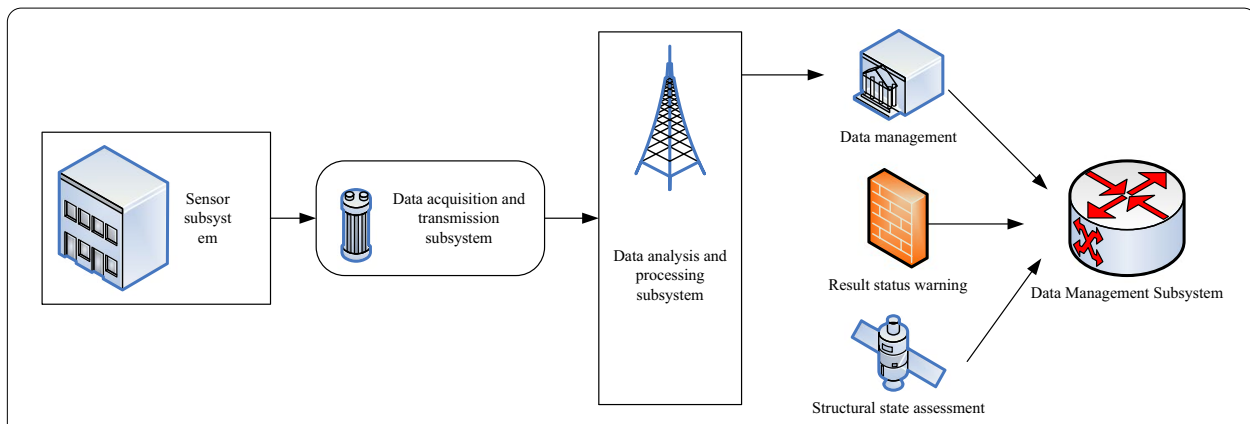

Fig. 2 Relationship between the sub-systems of the health monitoring system 


$$
p=\left[\psi_{\mathrm{x}} \psi_{x}\right] \psi S
$$

Among them, $S$ is the output of initially selected candidate measuring points; $\Psi x$ is the part of the modal matrix that is less on the candidate measuring points; $p$ is the modal coordinate vector. If the actual number of selected measuring points is $i$, then the problem can be expressed as how to find $i$ measuring points from the selected measuring points of $x$, and the modal linear independence measured by these $i$ points is modal space Best estimate. Here, the covariance of the error is taken as the best estimate. Considering the influence of noise, the output equation is rewritten as:

$$
S=\psi_{x} p+\mathrm{i}
$$

where $S$ is the output of the initially selected candidate measurement points, $\psi_{x}$ is the lesser part of the candidate measurement points in the modal matrix; $p$ is the modal coordinate vector, and the number of measurement points is $i$.

Then, the covariance of the estimation error is:

$$
Q=[(p-\hat{p})]=\psi_{s} \psi_{z}=P
$$

Among them is the information matrix. Assuming that the noise is Gaussian white noise, then:

$$
P=\frac{\psi_{m} \psi_{n}}{\varphi_{0}}=\frac{A}{\varphi_{0}^{2}}
$$

It can be seen from formula 6 that the minimum of the covariance $Q$ of the estimation error is equivalent to the maximum of the Fisher information matrix $P$, that is, the estimation of the trace of matrix $A$ or the maximum value of its determinant in formula 7 is an unbiased estimation. Matrix $A$ can be written as:

$$
A=\sum_{i=1}^{z}\left[\psi_{m}\right]\left[\psi_{n}\right]=\sum_{i=1}^{z} A^{i}
$$

Among them, $A^{i}$ represents the contribution of the first degree of freedom to the matrix. The characteristic equation of solution matrix $A$ is:

$$
\begin{aligned}
& (A-\lambda \mathrm{k}) \psi=0 \\
& \psi_{\mathrm{n}} A \psi=\lambda \psi_{\mathrm{n}} \psi=k \\
& \psi_{n} \lambda \psi=A^{-1}
\end{aligned}
$$

The construction matrix $E$ is:

$$
\begin{aligned}
& E=\psi \varphi \lambda\left(\psi_{m} \varphi\right)^{n} \\
& E=\psi_{n}\left(\psi_{m} \psi\right)^{-1} \psi^{n}
\end{aligned}
$$


Obviously in Eq. 12, $E$ is an idempotent matrix, its eigenvalue is 1 or 0 , and the trace is equal to the rank, that is:

$$
\operatorname{tr}(A)=\operatorname{rank}(A)
$$

The $m$ th element on the diagonal represents the contribution of the $m$ th degree of freedom or measurement point to the rank of the matrix $\Psi$, that is, the contribution to the matrix $A$. Write the diagonal elements of $E$ as a column vector as:

$$
E_{k}=\left[E_{11}, E_{22}, \ldots, E_{x x}\right]
$$

Among them, the size of each element represents the relative size of the contribution of each degree of freedom or measurement point to the rank of the matrix $\Psi$. According to the effective independence method, when the Fisher information matrix $P$ takes the maximum value. The covariance $Q$ of the estimation error is minimum, the norm selected here is:

$$
\|P\|=\left(\psi^{\mathrm{T}} \psi\right)_{2}
$$

The requirement of $P$ can be met by $\Psi$. According to the related matrix theory, QR decomposition of column principal elements is a simple and effective method when selecting a subset of matrix column vectors with the largest norm. Perform QR decomposition of column principal elements of $\Psi$, and select a subset of the column vector group as:

$$
\psi E=Q R=\left[\begin{array}{ccc}
R_{11} & R_{1 M} & R_{1 N} \\
\dot{0} & \cdot & \cdot \\
R_{M M} & R_{N N}
\end{array}\right]
$$

Among them, $E$ is the permutation matrix, and the first $M$ columns of the matrix $\Psi E$ correspond to a set of row vectors with a larger norm in the matrix $\Psi$. This $M$ measurable degree of freedom is a set of measurable degrees of freedom that makes the information matrix have a larger norm. Assuming a mode matrix $\Psi$ with $i$ th mode shape, the modal kinetic energy corresponding to the $k$ th mode of $i$ th test degree of freedom is defined as:

$$
M K E=\psi \sum_{\mathrm{j}=1} M \psi
$$

For a kinematic structure, which has $\mathrm{n}$ degrees of freedom, the strain energy matrix $E$ of each degree of freedom on the structure is defined as:

$$
E=\frac{1}{2} u K u=\frac{1}{2}(\psi p) K \psi p
$$

Among them, $\Psi$ is the normalized mode matrix; $u$ is the displacement matrix; $p$ is the normalized coordinate matrix; $K$ is the stiffness matrix, which is a symmetric matrix. The modal strain matrix is defined as: 


$$
E_{m s e}=\left[\begin{array}{ccc}
\sum \psi_{i 1} k_{i j} \psi_{j 1} & \sum \psi_{i 2} k_{i j} \psi_{j 2} & \sum \psi_{i n} k_{i j} \psi_{j n} \\
\sum \psi_{i 2} k_{i j} \psi_{j 1} & \sum \psi_{i 2} k_{i j} \psi_{j 2} & \sum \psi_{i 2} k_{i j} \psi_{j n} \\
\sum \psi_{i n} k_{i j} \psi_{j 1} & \sum \psi_{i n} k_{i j} \psi_{j n} & \sum \psi_{i n} k_{i j} \psi_{j n}
\end{array}\right]
$$

Among them, $k_{i j}$ is the connection coefficient between node $i$ and node $j ; \Psi_{i j}$ is the component of the $j$ th order matrix on the $i$ th degree of freedom, and the matrix $E$ is a symmetric matrix. The element in the $i$ th row and the $j$ column of the matrix represents the strain energy related to $i$ th and $j$ th modes; the sum of all the elements in $i$ th row represents the total modal strain energy related to $i$ th mode. Select $m$ from the $n$ degrees of freedom of the dynamic structure. The set of $m$ measuring points with the largest modal kinetic energy is used as the sensor configuration point.

Because the single intelligent optimization algorithm has different performance defects, the traditional optimization bit algorithm based on the single intelligent optimization algorithm also has defects, and the algorithm based on the single intelligent optimization algorithm also has shortcomings. The traditional optimization algorithm has fast convergence speed and high optimization accuracy, but it is easy to fall into the local optimum in the later stage, resulting in poor optimization results at individual times; the intelligent optimization algorithm has good stability and strong global optimization ability, and it is not easy to fall into the local optimum. However, its convergence speed is slower than traditional optimization algorithms, and the optimization accuracy is slightly lower than that of intelligent optimization algorithms.

\section{Experimental analysis validity of monitoring test indexes of healthy fitness cardio-lung track and field sensor}

\subsection{Comparison of different test methods for the same index of physical fitness test based} on sensor monitoring

Based on the research perspective of statistical pattern recognition, the healthy physical fitness cardiopulmonary track and field sensor in this paper is researched according to the research idea of statistical pattern recognition. The standard process of pattern recognition includes data collection, data processing, feature extraction and selection, classification and recognition, and human movement monitoring [30]. The system structure diagram of the healthy fitness cardiopulmonary track and field sensor is shown in Fig. 3.

The mobile terminal uses healthy fitness cardiopulmonary track and field sensors to collect human body data, calculates human body movements and health factors through recognition algorithms, uses its own health system to judge human body conditions, communicates with the server through a wireless network, and takes the form of an operation interface Provides the function of interacting with the server. The equipment used in this article to monitor sports health is an acceleration sensor and a photoelectric heart rate sensor. The acceleration sensor model is FEA-XX-YZZ-M1, dual axis, power supply voltage is $12 \mathrm{~V}$, input signal $4-20 \mathrm{~mA}$, frequency response is $0.5-20 \mathrm{~Hz}$. The photoelectric heart rate sensor selected is PluseSensor, the power supply voltage is $3.3-5 \mathrm{~V}$, the output signal range is $0-\mathrm{VCC}$, the detection signal is a light reflection signal, and the output signal type is an analog signal.

The server is mainly responsible for real-time monitoring and recording of the movement of the human body in the mobile terminal, and saves the information about human 


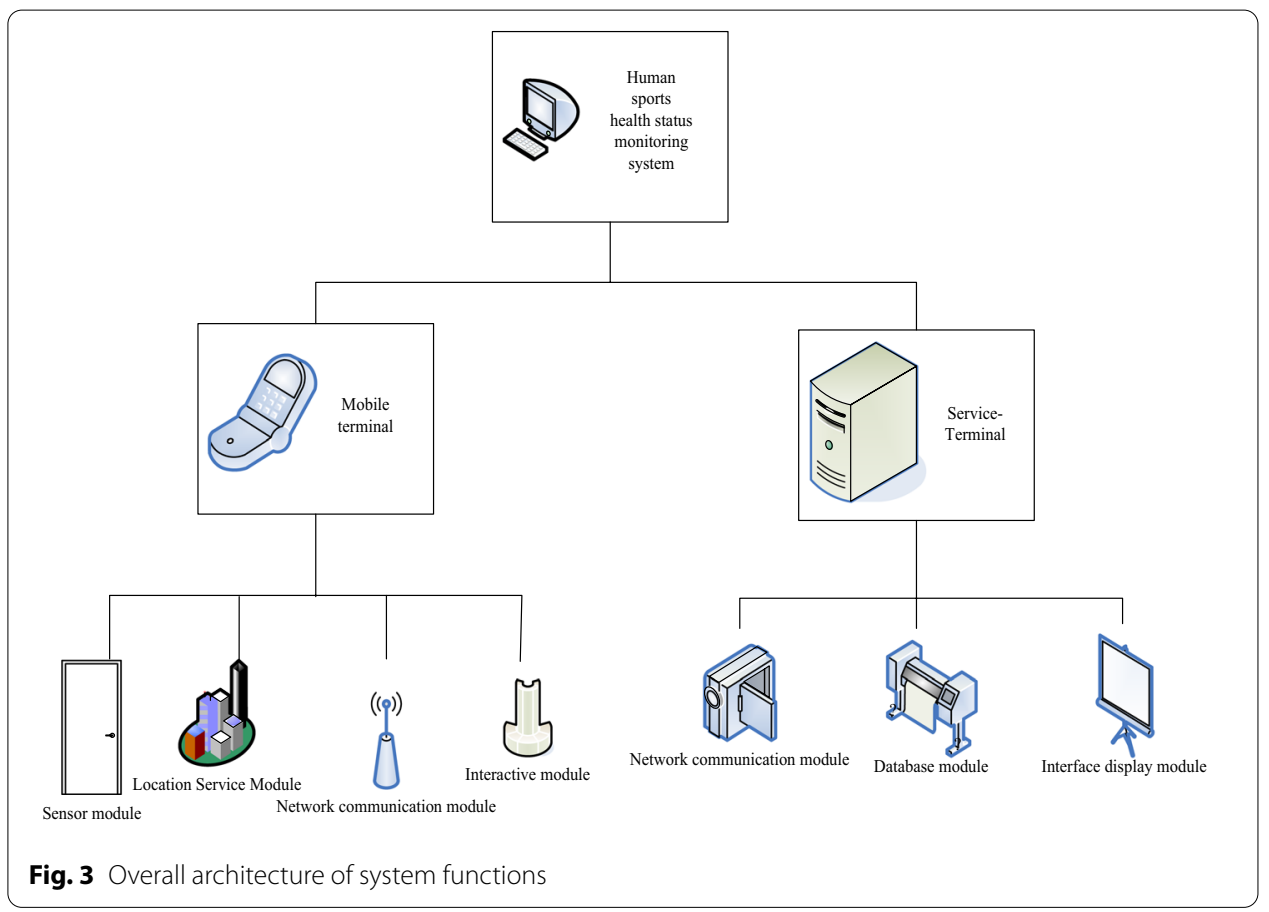

Table 1 Statistics of experimental test objects

\begin{tabular}{lll}
\hline Project & Male & Female \\
\hline Number of people (numbers) & 160 & 140 \\
Height $(\mathrm{cm})$ & $176.83 \pm 1.32$ & $160.3 \pm 1.16$ \\
Body weight $(\mathrm{kg})$ & $69.1 \pm 2.12$ & $52 \pm 1.63$ \\
\hline
\end{tabular}

movement and health in the database for historical query. This article takes the conventional indicators in the health fitness test at home and abroad as the main research object. The experimental test selects a university undergraduate junior student as the experimental object. The special item is not limited. A total of 300 students are selected, with an average age of 23-24 years old. Among them, 160 boys have an average height of $176.83 \mathrm{~cm}$ and an average weight of $69.1 \mathrm{~kg}$, and 140 girls have an average height of $160.3 \mathrm{~cm}$ and an average weight of $52 \mathrm{~kg}$. Statistics are shown in Table 1.

In order to ensure the accuracy of the test, the testers are called for rigorous professional training before the test. Each tester is only responsible for the test of one indicator. In order to minimize the measurement error, each indicator is performed six times per method. Repeat the measurement. The test indicators are the seven items in the wood-walking experiment of middle finger distance, calf length, upper arm relaxed circumference, upper arm tension circumference, abdominal sebum, sitting body forward bending, and static balance ability. Two different testing methods are used for the middle finger distance, calf length, upper arm relaxed circumference, and upper arm tension circumference. They are the prone position test method and the standing position test method. According to the internal and external factors that affect the human body's balance, the human body's standing balance can be divided 
into two categories. The external factors can be divided into the human body's motion state, the number of support points, the motion state of the support surface, and the surface hardness of the human body. Intrinsic factors can be divided into three subcategories: physiological factors, psychological factors, and technical factors. The test of human balance ability includes the support time test method, the force measurement platform pressure center test method, the direct measurement method of the center of gravity of the human body, and more index comprehensive test and other methods. The details are as follows:

Prone position test method: Adjust the measuring scale on the platform, the person separates the feet, raises the arms horizontally and extends as much as possible, puts the upper body on the balance scale, adjusts the zero with one fingertip, and extends the upper limb to one side on the other side. The palms, arms, and chest should be close to the ulnar surface, the arms should be in a straight line, and the tester should face the subject to measure and record the data.

Standing posture: Adjust the measuring ruler on the platform. The subject stands facing the wall, with both arms raised horizontally and extended as much as possible, the upper body is attached to the balance ruler, one fingertip is adjusted to zero, and the other side stretches the upper limb to one side. The palms, arms and chest should be close to the ulnar surface, the arms should be in a straight line, and the tester should face the subject to measure and record the data. The statistical results are shown in Table 2.

Table 2 shows that the $P$ values of middle finger distance, calf length, and upper arm tension circumference are greater than 0.05 , which are not statistically significant, indicating that there are no two different measurement methods for middle finger distance, calf length, upper arm tension circumference, prone position and standing position significant difference. However, the reliability coefficient of the middle finger distance in the prone position is 0.998 , which is greater than 0.926 in the standing position, and the standing position is slightly larger than in the prone position, so it is recommended to use the standing position to measure the middle finger distance. In the same way, calf circumference, upper arm relaxed circumference, and upper arm tension circumference are suitable for prone position measurement. The $P$ value of the two different measurement methods of the horizontal and vertical positions of the

Table 2 Experimental results of two test methods for middle finger distance, calf length, and upper arm tension

\begin{tabular}{lllll}
\hline Index & Testing method & $\boldsymbol{P}$ & Mean value (cm) & $\begin{array}{l}\text { Stability } \\
\text { coefficient } \\
(R)\end{array}$ \\
\hline Middle finger spacing & Prone position & 0.981 & 177.121 & 0.926 \\
& Standing position & & 177.263 & 0.998 \\
Calf length & Prone position & 0.923 & 38.04 & 0.999 \\
& Standing position & & 38.00 & 0.998 \\
Relax upper arm & Prone position & 0.000 & 27.523 & 0.997 \\
& Standing position & & 28.023 & 0.996 \\
Tight upper arm circumference & Prone position & 0.097 & 30.352 & 0.998 \\
& Standing position & & 30.304 & 0.995 \\
\hline
\end{tabular}


upper arm relaxation is less than 0.05 , indicating that the difference between the two different measurement methods is statistically significant. Therefore, we have reason to believe that the two measurement methods are significantly different.

Abdominal sebum, sitting forward bending, static balance ability are tested. Abdominal sebum uses horizontal pinching and vertical pinching. The horizontal (vertical) pinching of abdominal sebum refers to the use of the left thumb and index finger (about $3 \mathrm{~cm}$ apart). The examiner Elevate the skin and tissue to below the vertical index about $2 \mathrm{~cm}$ above the intersection of the horizontal line and the vertical line of the clavicle center. Hold the skinfold meter with your right hand and measure it $1 \mathrm{~cm}$ below the point. After the pointer of the indicator stops steadily, take the reading. The unit is recorded as millimeters. The sitting body forward test method with one leg and both legs, and the sitting body forward bend test method with one leg: sit down with your head and your legs in key positions, then extend your arms forward, bend your upper body forward and press down until the arm can no longer be extended. Take the heel as the side and press the nail size with both hands; the distance from the center point to the heel line is a negative number; the distance above the heel line is a positive number. The record is in centimeters, with one decimal place. The test method of the sitting position with the legs bent forward: Sit with the head and straighten the legs. The passenger presses the knees on the side of the head, and the heels are no more than $10 \mathrm{~cm}$. She leaned forward and pushed down until her arm could reach. Take the heel as the side and record the extension of the middle toe; the centimeter tip of the middle toe in the negative row of the heel; the distance above the heel line is a positive number. Keep one decimal place. The static balance adopts the horizontal wood treading method and the longitudinal wood treading method. Horizontal (longitudinal) stepping method: Arrange wooden slats on the floor, then test the steps on the front slats used to support the legs, create a cross with wooden slats (the same as the base of the wooden slats), and calculate from the wooden slats The other leg stops moving one leg until the moment it loses balance, and then the time is recorded in "seconds." The statistical results are shown in Table 3.

Table 3 shows that the $P$ value of the abdominal sebum thickness measured by the horizontal and vertical measurement methods and the static balance ability measured by the horizontal and vertical measurement methods is greater than 0.05 , and the two are not significant. Difference, but the reliability of the horizontal wood treading experiment

Table 3 Experimental results of two test methods for partial sebum, sitting body forward flexion, and static balance ability

\begin{tabular}{lllll}
\hline Index & Testing method & $\boldsymbol{P}$ & Mean value & $\begin{array}{l}\text { Stability } \\
\text { coefficient } \\
(R)\end{array}$ \\
\hline Abdominal sebum thickness & Horizontal pinch & 0.177 & $14.293 \mathrm{~mm}$ & 0.994 \\
& Vertical pinch & & $14.461 \mathrm{~mm}$ & 0.992 \\
Sitting forward bending & Single leg & 0.000 & $19.216 \mathrm{~cm}$ & 0.993 \\
& Legs & & $16.834 \mathrm{~cm}$ & 0.993 \\
Wood treading experiment & Horizontal & 0.932 & $51 \mathrm{~s}$ & 0.997 \\
& Vertical & & $49 \mathrm{~s}$ & 0.992 \\
\hline
\end{tabular}


is higher. By comparing the difference and stability of the two different test methods of sitting forward bending, it is concluded that there is a significant difference between the single-leg test and the two-legged test. The stability coefficient of the two methods is high and both are relatively stable.

\subsection{Research the rationality of the evaluation indexes of physical fitness cardiopulmonary endurance}

Cardiorespiratory endurance refers to the ability of the circulatory and respiratory systems to deliver oxygen to the body through continuous human activities. A person with good cardiorespiratory endurance has strong athletic ability because he can make good use of the oxygen entering the body, extend his training time, and make it easier for him to manage his daily activities. Current fitness tests in the USA include body composition (sebum size, body mass index), cardiorespiratory capacity (1600-m run), muscle endurance (sit-ups, pull-ups) and sensitivity (sit-ups). In Japan, it includes cardiorespiratory endurance (12 min of running, 3 min of stepping), muscle strength (grip strength, back strength, vertical jump), muscle endurance (stand-ups, repeated horizontal jumps), and sensitivity (standing forward bending). China's experiment includes body composition including height and weight, cardiovascular endurance (vital capacity, stepping test), muscle endurance (back strength, straight jump), muscle endurance (sit-ups, push-ups), and sensitivity (sitting forward bending) wait. We distributed a questionnaire survey report to 60 sports professionals in a university. The statistics of the questionnaire survey on the rationality of the "physical fitness" cardiorespiratory endurance test program for ordinary college students are shown in Fig. 4.

Figure 4 shows that the 2400-m jogging and 12-min walk are considered the most reasonable in the students' "physical fitness" cardiorespiratory endurance test item. Among them, 18 experts think that the 2400 jogging is very reasonable, and 28 experts think it is reasonable. For the 12-min run, 20 experts thought it was very reasonable, and 22 experts thought it was more reasonable; 46 experts thought it was more reasonable for the 1600-m run. The two sports that experts believe are the most

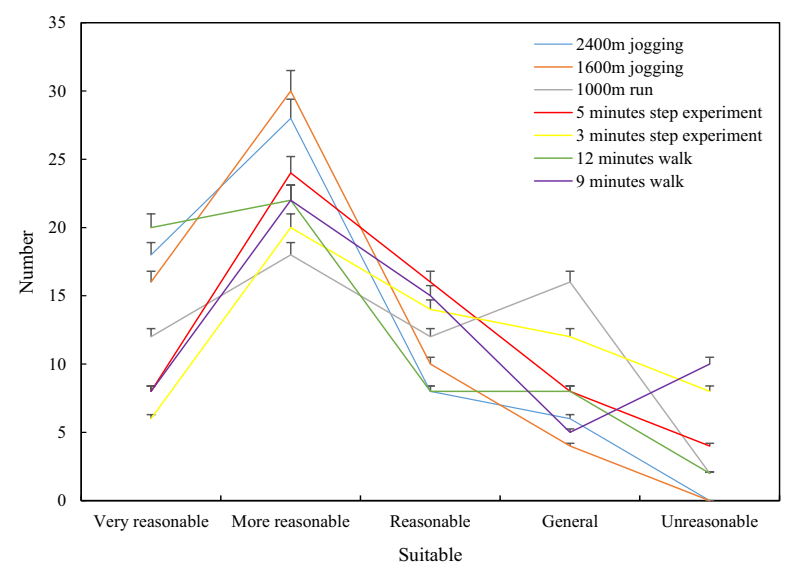

Fig. 4 Experts' statistics on the rationality of indicators in the "Physical Fitness" cardiopulmonary endurance test of students 


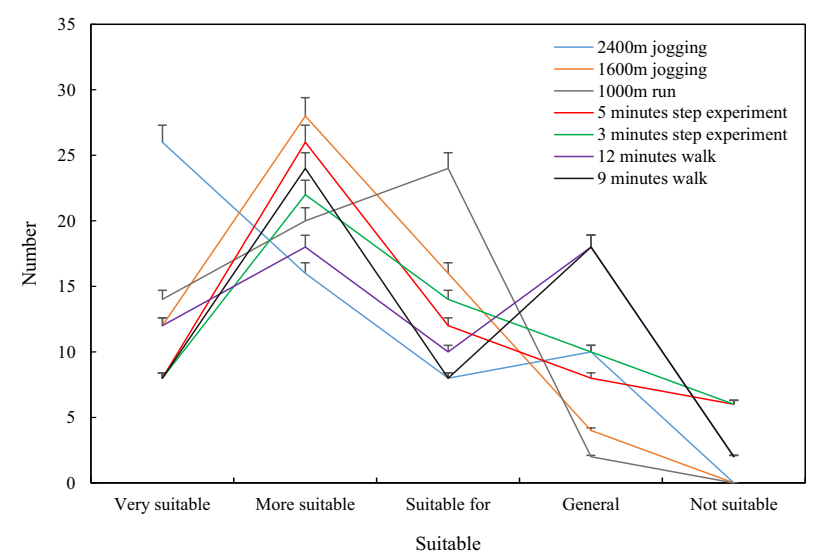

Fig. 5 Operability survey results of the physical fitness "cardiopulmonary endurance test project"
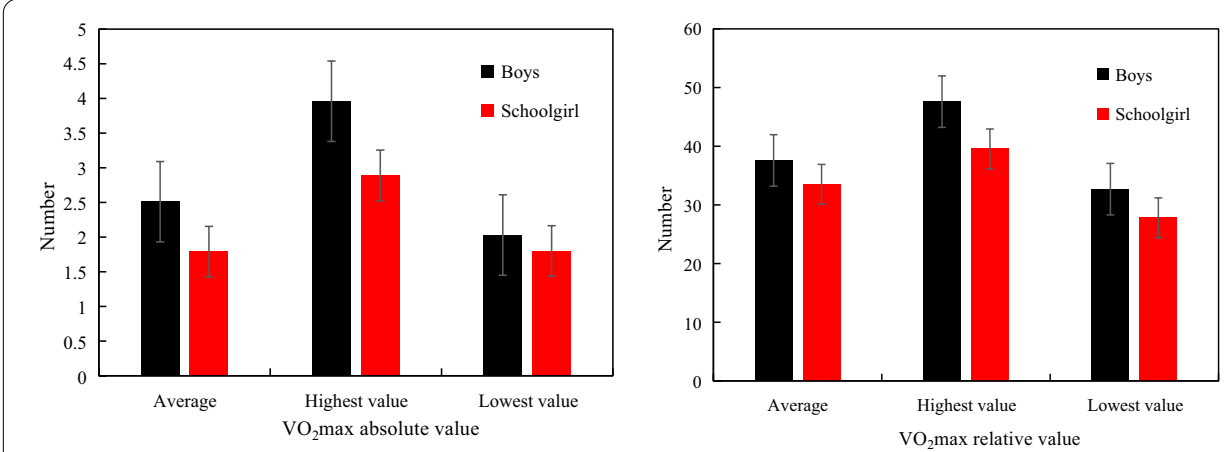

Fig. 6 List of basic conditions of the maximum oxygen consumption of college students

unreasonable are the 1000-m run and the 3-min step test. Among them, 16 experts think that the 1000-m run is fair, and 2 experts think it is unreasonable; the 3-min step test thinks that the average expert is 12 people. Eight people thought it unreasonable. The operability survey results of the physical fitness cardiopulmonary endurance test items are shown in Fig. 5.

Figure 5 shows that the expert group believes that the most practical items in the "Physical Fitness" cardiorespiratory endurance test for school students are the 2400-m run and the 1600-m run. Among them, 26 experts believe that the $2400-\mathrm{m}$ run is the most appropriate. Experts think it is more appropriate; 12 experts think that the 1600-m run is the most suitable, and 28 experts think it is more suitable. The test items that are considered the least operability in the university are the 12-min run and the 3-min step test. Among them, 18 people think that the 12-min run is generally operability, and two people think that the 12-min run is inappropriate. The most appropriate test index for the body's cardiorespiratory capacity and aerobic endurance level is the maximum oxygen consumption. In kinesiology and physiology, people usually use the maximum oxygen consumption to evaluate people's cardiovascular capabilities. By testing the maximum oxygen consumption of the test subjects, the test results of the maximum oxygen consumption of male and female college students are shown in Fig. 6. 
Figure 6 shows that the average absolute value of maximum oxygen consumption for boys is $2.51 \mathrm{~L} / \mathrm{min}$, the highest value is $3.96 \mathrm{~L} / \mathrm{min}$, and the lowest is $2.03 \mathrm{~L} / \mathrm{min}$. The average absolute value of maximum oxygen consumption for girls is $1.79 \mathrm{~L} / \mathrm{min}$. The highest value is $2.89 \mathrm{~L} / \mathrm{min}$, and the lowest value is $1.51 \mathrm{~L} / \mathrm{min}$. The average relative value of maximum oxygen consumption for boys is $37.58 \mathrm{ml} / \mathrm{kg} / \mathrm{min}$, the highest is $47.60 \mathrm{ml} /$ $\mathrm{kg} / \mathrm{min}$, and the lowest is $32.68 \mathrm{ml} / \mathrm{kg} / \mathrm{min}$. The average relative value of maximum oxygen consumption for girls is $33.51 \mathrm{ml} / \mathrm{kg} / \mathrm{min}$. The highest is $39.55 \mathrm{ml} / \mathrm{kg} / \mathrm{min}$, and the lowest is $27.80 \mathrm{ml} / \mathrm{kg} / \mathrm{min}$. The heart rates of boys at $1000 \mathrm{~m}$ and $2400 \mathrm{~m}$ are shown in Fig. 7.

Figure 7 shows that the average values of the heart rate at $1000 \mathrm{~m}$ and the heart rate at $2400 \mathrm{~m}$ are 134 beats/minute and 184 beats/minute respectively. According to the criterion of maximal oxygen uptake, the average value of boys completing $1000 \mathrm{~m}$ is less than the criterion of maximal oxygen uptake, while the average of $2400 \mathrm{~m}$ reaches the criterion of maximal oxygen uptake. Therefore, the 2400 -m cardiopulmonary test is more in line with the "physical fitness" cardiopulmonary endurance test indicators of ordinary college students. The heart rate of girls at $800 \mathrm{~m}$ and $2000 \mathrm{~m}$ is 131 beats/minute and 173 beats/minute, respectively. According to the maximum oxygen uptake criterion, the subject's heart rate is greater than 180 beats/minute. The $2000-\mathrm{m}$ test meets this standard, and $800 \mathrm{~m}$ did not meet the standard. Therefore, the $2000-\mathrm{m}$ endurance run can better reflect the cardiorespiratory endurance level of the students than the traditional 800-m run. For measuring the cardiorespiratory volume and heart rate of athletes after completing $2400 \mathrm{~m}, 12 \mathrm{~min}$, and $1000 \mathrm{~m}$, measuring these data can get the best training items and training volume, and it can be concluded that the teaching content of track and field elective courses requires fitness and guidance, not the traditional competitive teaching mode and teaching method based on technology teaching and skill transfer.

\subsection{Application of healthy physical fitness concept in track and field teaching}

In order to better formulate teaching prescriptions for track and field health and fitness, to understand students' views on track and field teaching, and to discover the current shortcomings in the teaching of track and field optional courses, a questionnaire survey was conducted on 200 students' attitudes and views on track and field teaching Fig. 8 shows.

Figure 8 shows that the overall satisfaction of students with the current track and field teaching is not high. $67 \%$ of the students think that the current track and field teaching
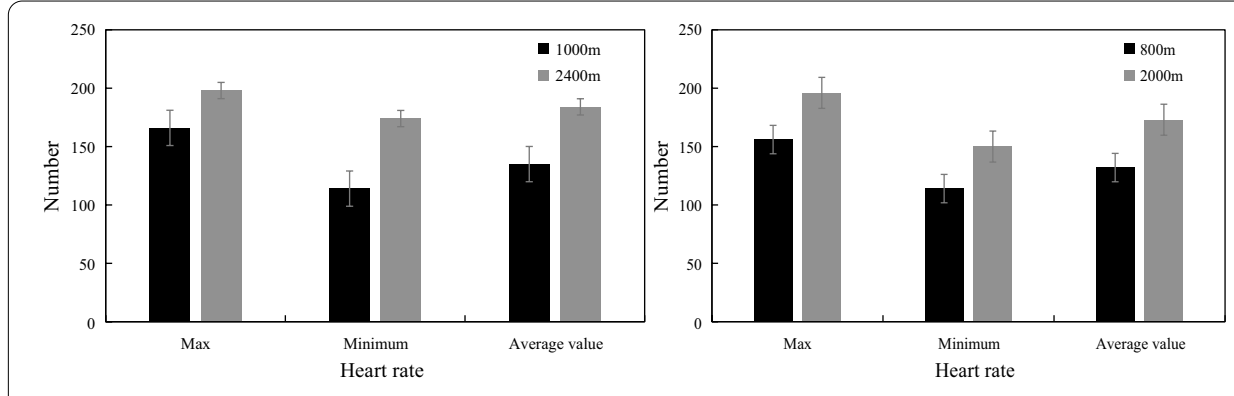

Fig. 7 A list of basic heart rates of boys and girls 

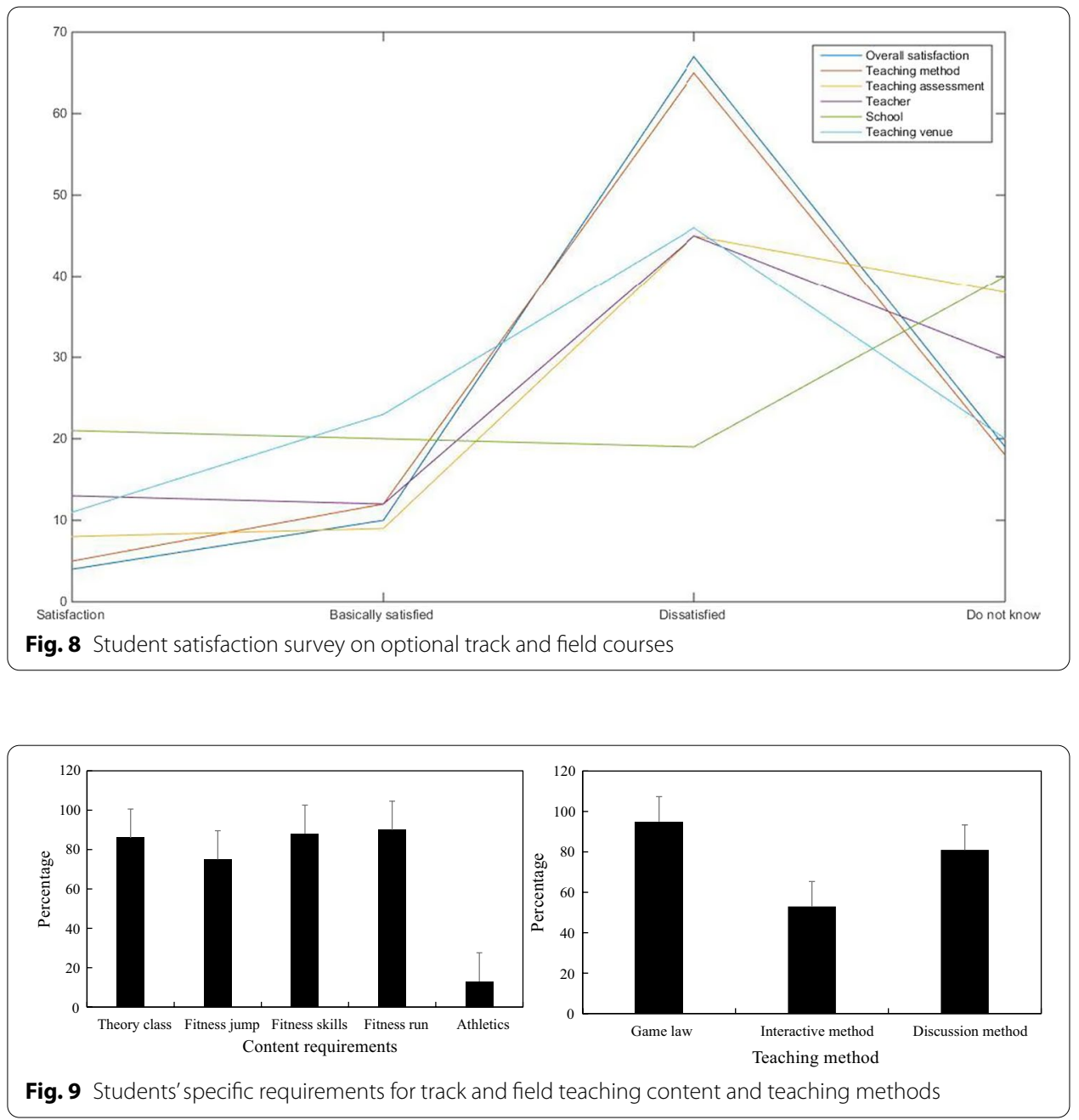

is not satisfactory. Among them, the teaching method and the teaching assessment are the most dissatisfied with $65 \%$ and $65 \%$, respectively. $45 \%$ of students are not satisfied. Student satisfaction is relatively high in the school's course time and equipment venues, which shows that the overall hardware equipment of the university is still up to the standard, and the course time is also guaranteed. The specific requirements of students on track and field teaching content and teaching methods are shown in Fig. 9.

Figure 9 shows that students have fitness and instructional requirements for the teaching content of the current path option courses, rather than the traditional competitive teaching mode that focuses on technical teaching and skill transfer. Students' requirements for track and field theory courses have reached $85 \%$, which shows that our track and field teaching has neglected to guide practice with theory. In the choice of practice course content, most students require more fitness-oriented fitness jumping, shooting, and running rather than competitive technical teaching. In terms of teaching methods, students prefer games and discussion teaching methods. $95 \%$ of students prefer gamification teaching methods, $81 \%$ of students prefer discussion teaching methods, and only $53 \%$ prefer interactive teaching methods. According to the students' views and requirements on track and field teaching, we have formulated relevant training programs based 


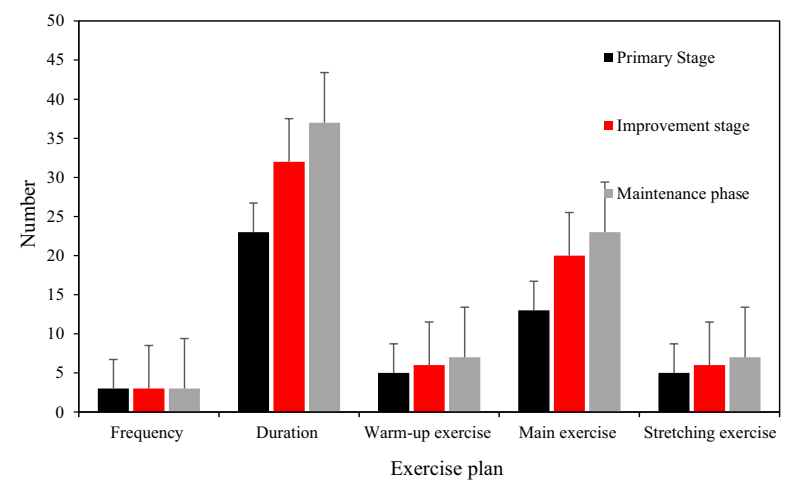

Fig. 10 Healthy physical fitness track and field teaching exercise plan
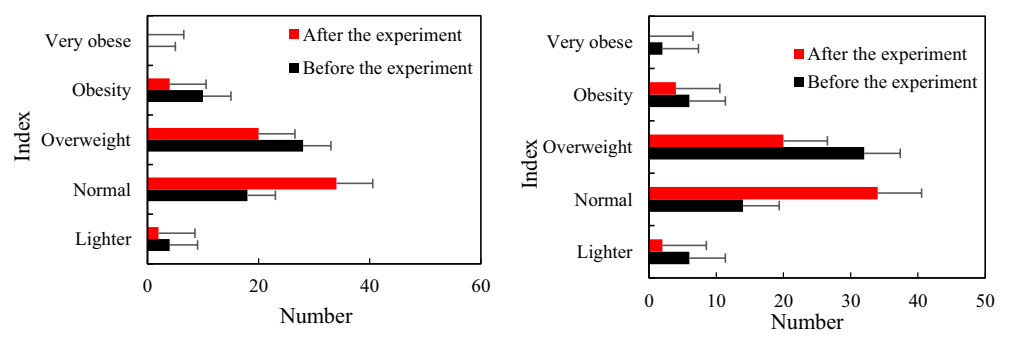

Fig. 11 BMI index statistics of students before and after the experiment

on fully reviewing relevant fitness prescription formulation principles and interviewing experts. Cardiopulmonary fitness aerobic exercise is mainly based on jogging, climbing and fitness. The specific program as shown in Fig. 10.

Figure 10 shows that the three stages require the weekly training channel to reach three times a week. The exercise duration is about $23 \mathrm{~min}$ in the primary stage, about $32 \mathrm{~min}$ in the improvement stage, and $37 \mathrm{~min}$ in the final maintenance stage. Among them, the warm-up exercise and post-run stretching are $5 \mathrm{~min}, 6 \mathrm{~min}$, and $7 \mathrm{~min}$ in the initial stage, improvement stage, and maintenance stage, respectively. After exercising for a period of time in accordance with this exercise program, test the impact of the new teaching method on the BMI index. The BMI index is the body mass index, which is the ratio of the weight/height square, and is currently used internationally to measure the degree of body fat and thinness. A standard of health is a reliable and appropriate evaluation index. The specific conditions of the BMI index of boys and girls before and after exercise are shown in Fig. 11.

From Fig. 11, we can know that there are still many problems with the physical quality of college students. Before the experiment, the number of boys or girls whose index was within the normal range was small, accounting for only about one-fourth of the statistics, and the number of people who were underweight, overweight, and obese was relatively large. After 4 months of teaching track and field health and fitness, the number of students whose index was in the normal range increased significantly. For example, the number of boys before the experiment increased to 34 after the experiment, and the number of girls who had 14 before the experiment increased to 34 people after the test. 
This shows that through professional athletic fitness training, the quality of students can be greatly improved.

\section{Discussion}

As the pace of life continues to accelerate, people physical and mental health problems have become more and more prominent. Obesity, cardiovascular disease and sub-health conditions caused by insufficient exercise have become more and more common, which has led to people's health and physical fitness increasing attention. Therefore, this article compares the validity of indicators in the health fitness cardiopulmonary track and field sensor monitoring test, and it can be found that:

(1) Vital capacity is one of the important indicators that directly reflect the changes in lung function after the human body participates in physical exercise, and it also reflects the changes in the exerciser's respiratory system from the side. Heart rate is the number of heart beats per minute. In aerobic exercise, heart rate is often used to control the intensity of exercise, assess physiological load, exercise degree, functional status and cardiac reserve function, etc. During the exercise process, the human body is due to the metabolism of muscle tissue involved in exercise exuberance increases the demand for oxygen. In order to adapt to this change, the body increases the blood running volume per unit time of the circulatory system. This value is expressed by cardiac output.

(2) The general process of healthy physical fitness cardiopulmonary track and field sensor's human motion monitoring according to statistical pattern recognition consists of data collection, data preprocessing, feature extraction and selection, classification and recognition. It can be seen from the experiment that in the experimental test of the two test methods of the same index, there are no significant differences in the test methods, including the distance between the middle finger, the length of the calf, the tight circumference of the upper arm, the abdominal sebum, and the balance ability, and the stability coefficient is large. The tester can freely choose the test method; but although there is no significant difference in the distance between the middle fingers, the stability coefficient of the standing position is significantly greater than that of the prone position. It is recommended to use the standing position test method to test the middle finger distance. In the two test methods of the same index, there are significant differences in the test results of upper arm relaxation and sitting body forward bending, but both have high stability. It is recommended that the tester choose the conventional test method.

(3) It can be seen that compared to a 12-min run, the 2400-m running test is easy to perform in ordinary colleges and universities. It only needs track and field or playground, and the test equipment is also very simple, only a stopwatch and a whistle are needed. There are no need for too many testers. A group of testers can have at least 15 people who will be tested. On the contrary, a 12-min walk is not easy to carry out in ordinary colleges and universities. It is necessary to measure the marks before the marking test and measure the distance after the competition. More equipment and more inspectors are needed, and there are fewer people who can test at a time. It is impossible to test more than a dozen people at a time. Based 
on the above analysis, in view of the actual development and practical performance of ordinary colleges and universities, the $2400-\mathrm{m}$ run is more suitable for school development than the 12-min walk. For this reason, it is recommended that college students use the 2400-m run for the "Physical Fitness Cardiopulmonary Endurance Test."

(4) The average heart rate of boys at $1000 \mathrm{~m}$ and $2400 \mathrm{~m}$ is 134 beats/minute and 184 beats/minute, respectively. According to the criterion of maximal oxygen uptake, the average value of boys completing $1000 \mathrm{~m}$ is less than the criterion of maximal oxygen uptake, while the average value of $2400 \mathrm{~m}$ reaches the criterion of maximal oxygen uptake. The heart rate of girls at $800 \mathrm{~m}$ and $2000 \mathrm{~m}$ is 131 beats/minute and 173 beats/minute, respectively. According to the maximum oxygen uptake criterion, the subject's heart rate is greater than180 beats/minute. The $2000 \mathrm{~m}$ test meets this standard, and $800 \mathrm{~m}$ did not meet the standard. Therefore, the long-distance endurance running can be more realistic and objective to evaluate the participants' cardiorespiratory endurance level than the short-distance endurance running.

(5) Healthy physical fitness is the basic physical ability of college students for work, study and leisure sports, and it is the guarantee of future work and the ability to improve work efficiency. From the perspective of the elements of healthy physical fitness, all content of relevant elements can be involved in track and field teaching content. In the cardiopulmonary endurance fitness level, you can improve the cardiorespiratory fitness level by slow walking or jogging; in the muscle endurance and speed fitness, it can be improved by fast running; as long as the track and field teaching content is selected reasonably, the teaching methods are correct, and the fitness is healthy. Almost all can be realized in track and field classroom teaching. Based on the theory of healthy physical fitness and track and field courses, most of the students' BMI index has been improved, tending to the normal level.

\section{Conclusion}

By using a healthy fitness cardiopulmonary track and field sensor to monitor the validity of the test indicators, we can find that through experiments, it can be seen that the distance between the middle finger, the length of the calf, the tight circumference of the upper arm, the abdominal sebum, and the stability coefficient of balance is relatively large, and there is no significant difference in the test method. Both test methods are acceptable. The conventional test methods are recommended for the tests of arm relaxation and forward bending in sitting position. At the same time, it is suggested that the current colleges and universities in our country should give priority to the endurance running project when choosing the "physical fitness" cardiorespiratory endurance test and evaluation index for ordinary college students. Choose the $2400 \mathrm{~m}$ run for boys and $2000 \mathrm{~m}$ run for girls as the test indicators of cardiorespiratory endurance. This is because the boys' $2400-\mathrm{m}$ run and the girls' 2000-m run are more convenient for running in school, and the heart rate standards and oxygen uptake are more suitable than sprints. In teaching students, students are currently not satisfied with the current track and field teaching, and believe that the teaching content of the track and field optional course 
requires fitness and guidance instead of the traditional competitive teaching mode and teaching method that focuses on technical teaching and skill transfer. In the above, students prefer the teaching methods of games and discussion, and the use of healthy physical fitness track and field teaching can effectively improve the health of college students. However, there are still shortcomings in the research. For example, in the cardiopulmonary measurement project, only the running project is measured, and the measurement project is not comprehensive enough, and I hope to realize it in the follow-up research.

\section{Authors' contributions}

JB involved in writing, editing, and data analysis. The author read and approved the final manuscript.

\section{Author's Information}

Jing Bao born in Yinchuan, Ningxia, People's Republic of China, in 1987. She received the Master's Degree from Xi'an Jiaotong University, People's Republic of China. Now, she works in the department of Physical education teaching and research, in Northwest University. Her research interests include health and physical fitness and Students' physical health.

\section{Funding}

The author(s) received no financial support for the research, authorship, and/or publication of this article.

\section{Availability of data and materials}

Data sharing does not apply to this article because no dataset was generated or analyzed during the current research period.

\section{Declarations}

Ethics approval and consent to participate

This article is ethical, and this research has been agreed.

\section{Consent for publication}

The picture materials quoted in this article have no copyright requirements, and the source has been indicated.

Competing interests

The authors declare that they have no competing interests.

\section{Author details}

${ }^{1}$ School of Public Policy and Administration, Xi'an Jiaotong University, Xi'an 710049, Shaanxi, China. ${ }^{2}$ Department of Physical Education Teaching and Research, Northwest University, Xi'an 710127, Shaanxi, China.

Received: 18 August 2021 Accepted: 20 December 2021

Published online: 14 January 2022

\section{References}

1. A.A. Alashab, K.A. Mohamad, Performance Management in Saudi Arabia: the effects of employee engagement, physical fitness and healthy lifestyle on job performance towards absenteeism. Int. J. Bus. Manag. 3(4), 01-14 (2020)

2. A. García-Hermoso, Y. Ezzatvar, J.F. López-Gil et al., Is adherence to the Mediterranean Diet associated with healthy habits and physical fitness? A systematic review and meta-analysis including 565,421 youths. Br. J. Nutr. 1(1), 31-36 (2020)

3. K. Rumi, Grip strength and healthy aging. J. Phys. Fit. Sports Med. Jpfsm Off. J. Jpn. Soc. Phys. Fit. Sports Med. 6(3), 145-149 (2017)

4. W. Chen, A. Hammond-Bennett, A. Hypnar et al., Health-related physical fitness and physical activity in elementary school students. BMC Public Health 18(1), 195-210 (2018)

5. P. Nelis, B. Schmitz, A. Klose et al., Correlation analysis of physical fitness and retinal microvasculature by OCT angiography in healthy adults. PLOS ONE 14(12), 769-772 (2019)

6. B.M. Antunes, F.E. Rossi, A.M. Teixeira et al., Short-time high-intensity exercise increases peripheral BDNF in a physical fitness-dependent way in healthy men. Eur. J. Sport Sci. 20(3), 1-8 (2019)

7. B. Dva, C. Sk, E. Pbwd et al., Physical activity, physical fitness and quality of life in outpatients with a psychotic disorder versus healthy matched controls in a low-income country. Schizophr. Res. 22(9), 1-2 (2021)

8. C. Song, L. Oxley et al., What determines irrigation efficiency when farmers face extreme weather events? A field survey of the major wheat producing regions in China. J. Integr. Agric. 17(08), 196-207 (2018)

9. N. Kasim, K.A. Taib, A. Maturidi et al., Debris flow numerical simulation model comparison with field events in Kuala Kubu Baru and Lentang, Malaysia. IOP Conf. Ser. Mater. Sci. Eng. 1051(1), 48-53 (2021)

10. L. Bernardo, K. Nadaoka, T. Nakamura et al., Island-enhanced cooling mechanism in typhoon events revealed by field observations and numerical simulations for a coral reef area, Sekisei Lagoon, Japan. Ocean Dyn. 67(11), 1-16 (2017) 
11. M. Muro, A. Mrowiec, F. Boix, Cytokine expression profile as predictive surrogate biomarkers for clinical events in the field of solid organ transplantation. Curr. Protein Pept. Sci. 18(3), 222-234 (2017)

12. E. Yaman, Physical and physiological correlates of social physique anxiety in college students. Univers. J. Educ. Res. 5(3), 334-337 (2017)

13. M. Pustiek, A. Kos, A. Kos et al., eQuilibrium: a prototype of a sensor-based balance training and monitoring system. Procedia Comput. Sci. 17(4), 340-346 (2020)

14. B. Barshan, A. Yurtman, Classifying daily and sports activities invariantly to the positioning of wearable motion sensor units. IEEE Internet Things J. 7(6), 4801-4815 (2020)

15. H.L. Mjøsund, E. Boyle, P. Kjaer et al., Clinically acceptable agreement between the ViMove wireless motion sensor system and the Vicon motion capture system when measuring lumbar region inclination motion in the sagittal and coronal planes. BMC Musculoskelet. Disord. 18(1), 124-126 (2017)

16. S.Y. Jeong, J.U. Lee, S.M. Hong et al., Highly skin-conformal laser-induced graphene-based human motion monitoring sensor. Nanomaterials 11(4), 951-963 (2021)

17. R.K. Kanna, R. Vasuki, Monitoring and analysis of coma patients using variable motion sensor system. Drug Invent. Today 11(6), 26-34 (2019)

18. A. Zaffaroni, W.T. Mcnicholas, S.J. Crinion et al., Ambulatory detection of sleep apnea using a noncontact biomotion sensor. J. Sleep Res. 29(9), 236-245 (2019)

19. R. Usamentiaga, D.F. García, Infrared thermography sensor for temperature and speed measurement of moving material. Sensors 17(5), 268-275 (2017)

20. Z. Zhang, Y. Wang, K. Miao et al., Design of wireless motion sensor node. J. Phys Conf. Ser. 1792(1), 14-21 (2021)

21. A. Andreychenko, A. Raaijmakers, A. Sbrizzi et al., Thermal noise variance of a receive radiofrequency coil as a respiratory motion sensor. Magn. Reson. Med. 77(1), 263-268 (2017)

22. D. Shin, T. Seong, J. Choi et al., Self-sustaining water-motion sensor platform for continuous monitoring of frequency and amplitude dynamics. Nano Energy 35(2), 179-188 (2017)

23. X. Zhang, Q. Gao, Q. Gao et al., Triboelectric rotary motion sensor for industrial-grade speed and angle monitoring Sensors 21(5), 1713-1732 (2021)

24. A. Anand, K. Patwardhan, R.N. Singh et al., Effects of Pranayama on mental health and physical fitness in healthy University students. Yoga Mimamsa 50(1), 27-30 (2018)

25. K. Segaert, S. Lucas, C.V. Burley et al., Fit to speak — physical fitness is associated with reduced language decline in healthy ageing. Sci. Rep. 8(1), 125-136 (2018)

26. S. Xie, Z. Yu, Z. Lv, Multi-disease prediction based on deep learning: a survey. Comput. Model. Eng. Sci. 128(2), 489-522 (2021)

27. M. Hu, Y. Zhong, S. Xie, H. Lv, Z. Lv, Fuzzy system based medical image processing for brain disease prediction. Front. Neurosci. 15, 714318 (2021)

28. M.N.K. Boulos, Z. Lu, P. Guerrero, C. Jennett, A. Steed, From urban planning and emergency training to Pokémon Go: applications of virtual reality GIS (VRGIS) and augmented reality GIS (ARGIS) in personal, public and environmental health. Int. J. Health Geogr. 16, 1-11 (2017)

29. Z. Cai, Xu. Zheng, A private and efficient mechanism for data uploading in smart cyber-physical systems. IEEE Trans. Netw. Sci. Eng. (TNSE). 7(2), 766-775 (2020)

30. Z. Lv, L. Qiao, Analysis of healthcare big data. Future Gener. Comput. Syst. 109, 103-110 (2020)

\section{Publisher's Note}

Springer Nature remains neutral with regard to jurisdictional claims in published maps and institutional affiliations.

\section{Submit your manuscript to a SpringerOpen ${ }^{\circ}$ journal and benefit from:}

- Convenient online submission

- Rigorous peer review

- Open access: articles freely available online

- High visibility within the field

Retaining the copyright to your article

Submit your next manuscript at $\gg$ springeropen.com 